\title{
48. THE EVOLUTION OF COMETARY NUCLEI
}

\author{
L. M. SHUL'MAN \\ Main Astronomical Observatory, Ukrainian Academy of Sciences, Kiev, U.S.S.R.
}

\begin{abstract}
The evolution of a cometary nucleus depends on the size distribution of the dust content. If all the dust particles are small enough, they will all be thrown off into the coma; the secular decrease in the comet's brightness is due simply to the decrease in the nuclear radius, and the comet eventually disintegrates. On the other hand, if larger grains are present, they will start to form spots of a low-emissivity mineral envelope on the surface of the nucleus; in this case, the decrease in brightness is due to the increase in the area of the screened part of the surface, and the reduction in the nuclear radius is comparatively small. It is shown that after about $90 \%$ of the surface has been screened, the rate of screening increases considerably, as smaller particles become trapped between the larger ones; and there will be a corresponding acceleration in the brightness decrease. These nuclei are eventually covered with a solid mineral envelope, and they are transformed into asteroids.
\end{abstract}

\section{Introduction}

The icy model for a cometary nucleus is now generally accepted. However, some of the conceptions based on this model deserve criticism. The first one is the supposition that a so-called old comet has a nucleus covered by a continuous envelope of dust and stones. The second is the idea that the mineral envelope can be suddenly thrown off when the internal gas pressure reaches a certain level.

It will be shown in the present paper that comets that do not have exposed ice on their nuclear surfaces would be observed, not as comets, but as asteroids. A new modification of the icy model will be proposed: an icy surface that either becomes slowly covered by spots of a mineral envelope (spotted model) or remains homogeneous, depending on the size of the grains impregnated in the ice.

The merging of the separate islands of nonvolatile matter into one continuous envelope results in the 'death' of the comet, just as the total disintegration of a dustless nucleus does.

\section{Effect of Grain Size}

We assume that in its initial state the surface layer of a nucleus is a homogeneous mixture of ices, grains, and stones. The granulometric composition of the mineral bodies is given by the distribution function

$$
F(a)= \begin{cases}n_{d} \frac{S a_{\max }^{s} a_{\min }^{s}}{a_{\max }^{s}-a_{\min }^{s}} \frac{1}{a^{s+1}} ; & a_{\min }<a<a_{\max } \\ 0 ; & a>a_{\max }, a<a_{\min }\end{cases}
$$

where $a$ is the radius of a grain and $n_{d}$ the number density of grains. From meteoric data we adopt $s=3$. A grain is in equilibrium at the nuclear surface if its radius satisfies the relationship 


$$
a_{\mathrm{eq}}=\frac{9}{32 \pi} \frac{C_{D} m n v^{2}}{G \rho_{d} \rho_{n} R}
$$

where $m, n$ and $v$ are the molecular mass, number density, and velocity of the gas, respectively, $\rho_{d}$ and $\rho_{n}$ are the mean mass densities of a grain and the nucleus, $R$ is the radius of the nucleus, $G$ is the gravitational constant, and $C_{D}$ is a drag coefficient given by the relation

$$
C_{D}=\frac{2}{3 S} \sqrt{\frac{\pi T_{d}}{T}}+\frac{2 S^{2}+1}{S^{3} \sqrt{\pi}} e^{-S^{2}}+\frac{4 S^{4}+4 S^{2}-1}{2 S^{4}} \frac{2}{\sqrt{\pi}} \int_{0}^{S} e^{-x^{2}} \mathrm{~d} x,
$$

where $T_{d}$ and $T$ are the temperatures of the grain and gas, and

$$
S=\frac{v}{\sqrt{(2 k T) / m}} \approx 0.35
$$

( $k$ being the Boltzmann constant) is a dimensionless parameter. Under typical conditions $C_{D} \approx 10$. All the grains with radii $a>a_{\mathrm{eq}}$ will be stored at the surface and will form the nonvolatile envelope.

Three cases are possible. If

$$
a_{\text {eq }}>a_{\text {max, }}
$$

then all the dust particles can be thrown off into the atmosphere. Thus the nucleus evolves in a uniform manner, the composition of the surface layer remaining homogeneous. In this case, the radius decreases, and the evolution finishes with the total disintegration of the nucleus. Dustless comets, such as P/Encke (Whipple and DouglasHamilton, 1966), have the same uniform evolution. A second possibility is

$$
a_{\mathrm{min}}<a_{\mathrm{eq}}<a_{\max }
$$

in which case the grains with radii $a_{\mathrm{eq}}<a<a_{\max }$ will be stored on the surface, and the surface layer becomes nonuniform. This is the spotted model. The dusty crust grows until it covers the whole surface and the comet turns into an asteroid. The third case, $a_{\mathrm{eq}}<a_{\mathrm{min}}$, is similar to the second. It differs only by absence of dust in the cometary atmosphere, except for the dust formed by condensation of carbon (Dolginov, 1967).

\section{Secular Decrease in Brightness}

The second of the above-mentioned cases is the most general one. To treat it in detail we shall use a semiempirical approach, taking for the gas flux the expression

$$
n v=n_{1} v_{1} / r^{n},
$$

which corresponds to the empirical law of brightness variation

$$
E=E_{1} / r^{n}
$$

$r$ being the heliocentric distance, and where quantities with subscript 1 refer to $r=1$ AU. Since $v$ changes slowly one can obtain 


$$
\begin{aligned}
& \left(\rho_{n} \sim \rho_{d} \sim 1 \mathrm{~g} \mathrm{~cm}^{-3}, \quad v_{1} \sim 10^{4} \mathrm{~cm} \mathrm{~s}^{-1}, \quad C_{D} \sim 10, \quad G \sim 6.7 \times 10^{-8},\right. \\
& \left.n_{1} \sim 10^{12} \mathrm{~cm}^{-3}, \quad m \sim 5 \times 10^{-23} \mathrm{~g}\right) \\
& a_{\mathrm{eq}}[\mathrm{cm}]=\frac{0.7}{R[\mathrm{~km}] r^{n}[\mathrm{AU}]}
\end{aligned}
$$

Below we shall need the following averaged quantities:

$$
\begin{aligned}
& \left\langle a^{2}\right\rangle=\frac{1}{n_{d}} \int_{a_{\min }}^{a_{\max }} a^{2} F(a) \mathrm{d} a \sim 3 a_{\mathrm{min}}^{2} \\
& \left\langle a^{3}\right\rangle=\frac{1}{n_{d}} \int_{a_{\min }}^{a_{\max }} a^{3} F(a) \mathrm{d} a \sim 3 a_{\mathrm{min}}^{3} \ln \frac{a_{\mathrm{max}}}{a_{\mathrm{min}}} \\
& \left\langle a_{s}^{2}\right\rangle=\frac{1}{n_{d}} \int_{a_{\mathrm{eq}}}^{a_{\max }} a^{2} F(a) \mathrm{d} a \sim \frac{3 a_{\mathrm{min}}^{3}}{a_{\mathrm{eq}}}\left(1-\frac{a_{\mathrm{eq}}}{a_{\mathrm{max}}}\right) .
\end{aligned}
$$

Instead of the number density $n_{d}$ we can use the fraction by volume $f_{v}$ of the dust. Obviously,

$$
f_{v}=n_{d} \pi a_{\min }^{3} \ln \frac{a_{\max }}{a_{\min }}
$$

If $\xi$ is the fraction of the surface screened by the crust, then the rate at which the radius decreases is defined by the relation

$$
\frac{\mathrm{d} R}{\mathrm{~d} t}=\frac{(1-\xi) m n_{1} v_{1}}{\left(1-f_{v}\right) \rho_{n} r^{n}}
$$

and thus the rate of screening is

$$
\frac{\mathrm{d} \xi}{\mathrm{d} t}=\pi\left\langle a_{s}^{2}\right\rangle n_{d} \frac{4 \pi R^{2}(\mathrm{~d} R / \mathrm{d} t)}{4 \pi R^{2}}=\frac{3 f_{v}(1-\xi) m n_{1} v_{1}\left[1-\left(a_{\mathrm{eq}} / a_{\max }\right)\right]}{4\left(1-f_{v}\right) \rho_{n} a_{\mathrm{eq}} r^{n} \ln \left(a_{\mathrm{max}} / a_{\min }\right)} .
$$

Substituting here the value of $a_{\mathrm{eq}}$ at perihelion and integrating over one revolution, we obtain the change in $\xi$ per revolution from

$$
\begin{array}{r}
\Delta \ln (1-\xi)=-2.8 \times 10^{5} \frac{R[\mathrm{~km}] \rho_{n}\left[\mathrm{~g} \mathrm{~cm}^{-3}\right]}{C_{D} v^{(p)}\left[\mathrm{cm} \mathrm{s}^{-1}\right]} \frac{f_{v}}{1-f_{v}} \frac{1-a_{\mathrm{eq}}^{(p)} / a_{\max }}{\ln \left(a_{\max } / a_{\min }\right)} \\
\times \frac{q^{3 / 2}[\mathrm{AU}]}{(1+e)^{n-1.5}} \int^{2 \pi}(1+e \cos \theta)^{n-2} \mathrm{~d} \theta
\end{array}
$$

where $v^{(p)}$ and $a_{\mathrm{eq}}^{(p)}$ are the values of gas velocity and equilibrium grain radius at perihelion, and $q, e$, and $\theta$ are the perihelion distance, orbital eccentricity, and true anomaly of the comet.

Finally, one can obtain an expression for the secular variation in the total absolute magnitude $H_{y}$ : 


$$
H_{y}=H_{y}^{(0)}+2.5 M \nu \Delta \ln (1-\xi),
$$

where $M$ is the modulus of common logarithms, and the decrease in absolute brightness changes linearly with the number of revolutions $\nu$. Such behaviour will take place until the particles with radii $a>a_{\mathrm{eq}}$ touch one another at the surface of nucleus. It is easy to show that when the particles merge approximately $\pi / 2 \sqrt{3} \simeq 0.9$ of the total area will be screened.

At this moment the second layer of the crust will start forming. Of course, the smaller particles will be trapped in the pores between the particles stored in the first layer. This is equivalent to the rapid decrease of the quantity $a_{\mathrm{eq}}$ to the value

$$
a_{\mathrm{eq}}^{\prime}=\left(\frac{2}{\sqrt{3}}-1\right) a_{\mathrm{eq}} \sim 0.15 a_{\mathrm{eq}},
$$

and the rate of brightness decrease will thus be multiplied by a factor of approximately 7. During the forming of the second layer of the crust the following relation holds:

$$
H_{y}=H_{y}^{(0)}+2.5 M \nu_{1} \Delta \ln (1-\xi)+16.5 M\left(\nu-\nu_{1}\right) \Delta \ln (1-\xi),
$$

where $\nu_{1}$ is the number of revolutions before the formation of the first layer of the crust.

The straight lines in Figure 1 show the idealized decrease in brightness as the crust formation proceeds layer by layer. In fact, of course, the brightness variation will be smoothed out, as shown by the broken curve, for one should take into consideration that the crust formation is controlled by the actual distribution of the grains in the ice. We are dealing with a stochastic phenomenon. In reality, separate islands of crust

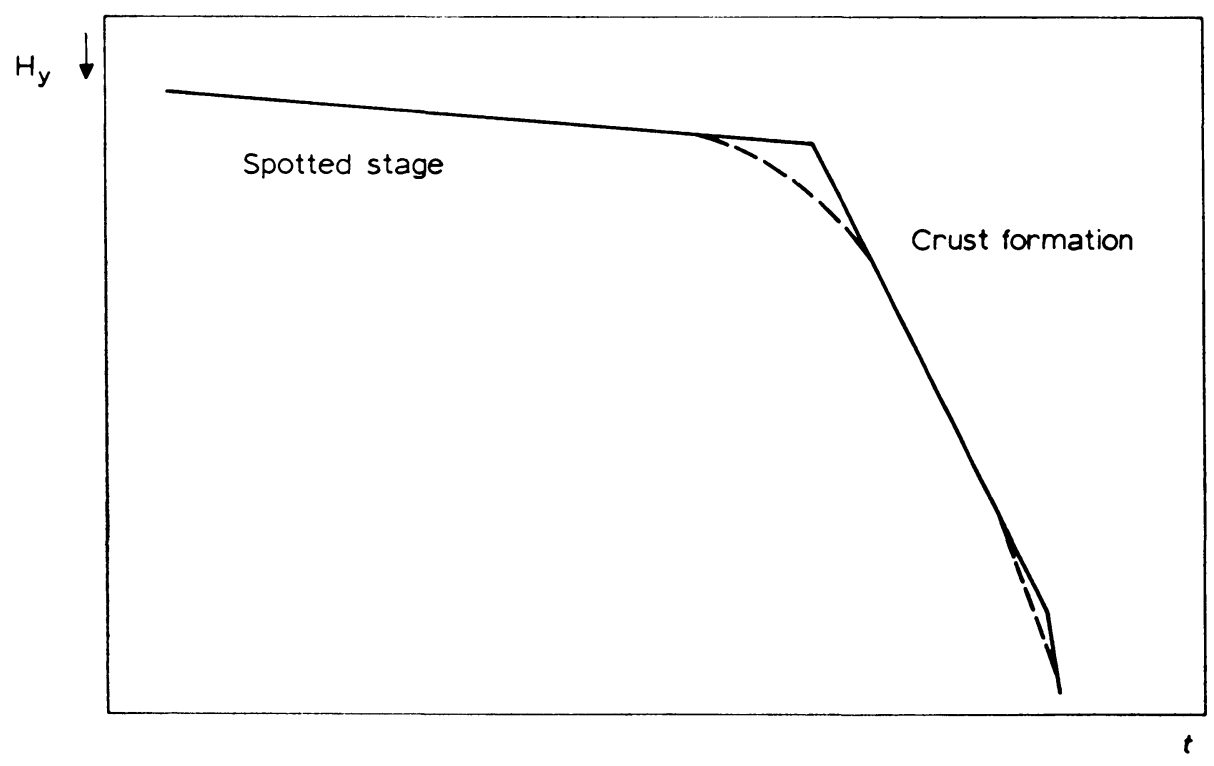

Fig. 1. Decrease in a comet's absolute brightness with time. 
are being formed. On some portions of the surface, only the first layer is being filled, while elsewhere the formation of the second layer has already begun. The picture resembles the thawing of dirty snow in spring.

\section{Invisibility of Completely Crusted Comets}

Now we shall show that a comet whose nucleus is shielded with a continuous crust would be invisible. The gas flow through the crust is controlled by the combined effect of sublimation, described by the isotherm

$$
n_{h} k T_{h}=10^{A-B / T_{h}},
$$

the energy balance at depth $h$

$$
n v \frac{k B}{M} \approx K \frac{T-T_{h}}{h},
$$

and diffusion

$$
n v \approx D \frac{n_{h}-n}{h}
$$

For rough estimates we do not need more precise forms for these equations. The subscript $h$ refers to the values at the depth $h$ (the thickness of the crust). $A$ and $B$ are the constants of the ice, and $K$ and $D$ are the coefficients of thermal conductivity and diffusion, respectively. The diffusion coefficient

$$
D \approx \beta \bar{v} a_{\mathrm{min}},
$$

where the dimensionless constant $\beta \leqslant 0$. . Since $n \ll n_{h}$ and $\bar{v} \sim v \sim \sqrt{k T / m}$, Equation (22) can be reduced to the form

$$
n \approx \beta n_{h} \frac{a_{\mathrm{min}}}{h}
$$

From Equations (20), (21), and (22) we can obtain the iterative formula

$$
T_{h}=B\left\{A-\log _{10}\left[T_{h}\left(T-T_{h}\right)\right]-\log _{10}\left[\frac{K M}{\beta a_{\min } B} \sqrt{\frac{m}{k T}}\right]\right\}^{-1} .
$$

We shall adopt the values $\beta \approx 0.1, a_{\min } \sim 10^{-6} \mathrm{~cm}, A=12, B=1400, m \sim 5 \times 10^{-23} \mathrm{~g}$. Following Whipple, we assume that the thermal conductivity is solely radiative, i.e., $K \sim 4 a_{\mathrm{min}} \sigma\left\langle T^{3}\right\rangle \sim 10^{-5}$. Thus we obtain $T_{h} \sim 120 \mathrm{~K}$; and for $h=10 \mathrm{~cm}, n v \simeq 6 \times 10^{8}$ $\mathrm{cm}^{-2} \mathrm{~s}^{-1}$. It is obvious that such a comet is much fainter than the comets that are usually observed. The dusty crust would make a comet initially of absolute magnitude 6 completely invisible.

Another property of comets with crusts is the thermal inertia of the shielding layer. The time scale of the thermal inertia is

$$
t_{i} \sim\left(\rho c h^{2} / K\right)
$$

where $\rho$ and $c$ are the density and specific heat of the crust. Under the conditions assumed above $t_{i} \sim 10^{11} \mathrm{~s}$. 
If the thermal conductivity coefficient is much higher than assumed here, the gas pressure at the icy surface can exceed the weight of the crust. But it would be wrong to conclude that the crust would then be thrown off. In fact, in this case we shall obtain a fluidized layer, possibly with channel structure. The fluidization does not prevent particles of the appropriate size from accumulating and the crust from growing. What happens is that the fluidization destroys contacts between grains, and thus the thermal conductivity decreases to its radiation value.

\section{References}

Dolginov, A. Z.: 1967, Astron. Zh. 44, 434.

Whipple, F. L. and Douglas-Hamilton, D. H. : 1966, Mem. Soc. Roy. Sci. Liège Ser. $512,469$. 